\title{
Chemical Compound Chemical Treatment in Animal Husbandry
}

\author{
Xiaoling Tang $\mathbb{D}^{1}$ and Ting Peng ${ }^{2}$ \\ ${ }^{1}$ Hunan Vocational College of Environmental Biological Technology, Hengyang 421005, Hunan, China \\ ${ }^{2}$ Department of Chemistry, Zhejiang Xianju High School, Taizhou 317300, Zhejiang, China \\ Correspondence should be addressed to Xiaoling Tang; tangxiaoling@hnebp.edu.cn
}

Received 6 August 2020; Revised 9 September 2020; Accepted 21 September 2020; Published 20 November 2020

Academic Editor: Tifeng Jiao

Copyright (C) 2020 Xiaoling Tang and Ting Peng. This is an open access article distributed under the Creative Commons Attribution License, which permits unrestricted use, distribution, and reproduction in any medium, provided the original work is properly cited.

\begin{abstract}
The acidulant is widely used in the production of animal husbandry, and its use is affected by many factors, including environmental factors, dosage, diet composition, and animal's own factors, so only the correct use of the acidulant can bring good results in animal production and financial income. This article takes acidifier as an example to study the application of compound chemical treatment in livestock farms. In this paper, the effect of using acidulant in the first 1 to 3 weeks after early weaning of piglets is obvious through this experimental study. The effect gradually decreases after 3 weeks and basically has no effect after 4 weeks. Experimental studies have found that the combination of organic acids, antibiotics, and high copper is the most effective. These three have different functions and have complementary or additive effects. Under harsh feeding conditions, especially when the environmental sanitation and environmental conditions are relatively poor, the effect of acidulants is better than good feeding conditions. Experimental data show that fulvic acid depletes milk's somatic cells in a short period of time and then quickly activates immune function, which is indicated by the increase in lymphocytes in the blood. When a large number of somatic cells migrate to the breast, the somatic cells in milk will also increase, thereby improving the immunity mediated by human cells. The experimental results show that the BFA formula added $1 \%$ to the cattle feed. After the research control of this experiment, the milk output increased by $9-17 \%$, and the quality milk output increased by $19.12 \%$, so the use of acidulant increased feed compensation and reduces gastrointestinal diseases and the reproduction of microorganisms in the rumen of dairy cows.
\end{abstract}

\section{Introduction}

Acidifier is organic or inorganic acid material, and it can be added to animal feed or drinking water alone or mixed, to inhibit the growth and reproduction of some harmful microorganisms, reduce the oxidative odor of feed, and can improve metabolism and certain human functions. In recent years, acidifiers have been widely used in the production of livestock feed and play an important role in improving the production efficiency of livestock. However, the environmental factors, dosage, dietary composition, animal type, age, weight, physiological stage, and other factors are greatly affected, so understanding the correct use and precautions of acidifiers requires careful study.

Biochemical fulvic acid is simulated natural humic acid production environment. According to specific biological oxidation reaction, organic medium was inoculated with various microbial strains to rapidly form BFA preparation, which is another environment-friendly biochemical product of the local Luyuan Animal Husbandry Company $[1,2]$. Its effective components in fulvic acid, DNA, are a kind of multivalent phenol-type polycondensate with nitrogen compounds, and aromatic compounds, containing phenol hydroxyl, hydroxyl, hydroxyl of alcohol, alcohol, acid, enol base, sulfonic acid, amino, free Ti hydrogen, half Ti base, and $\mathrm{Ti}$ base, such as oxygen- and carbon-based functional groups, have the high cation exchange capacity, integration capability, buffering capacity, south catalysis, and adsorption capability and can be directly involved in the tissue of redox process, greatly improving the activity of enzymes in the body; biochemical fulvic acid has been used in the development of animal husbandry, aquaculture, agriculture, and medicine. New concepts were introduced in the field of nutrition and immunology $[3,4]$. 


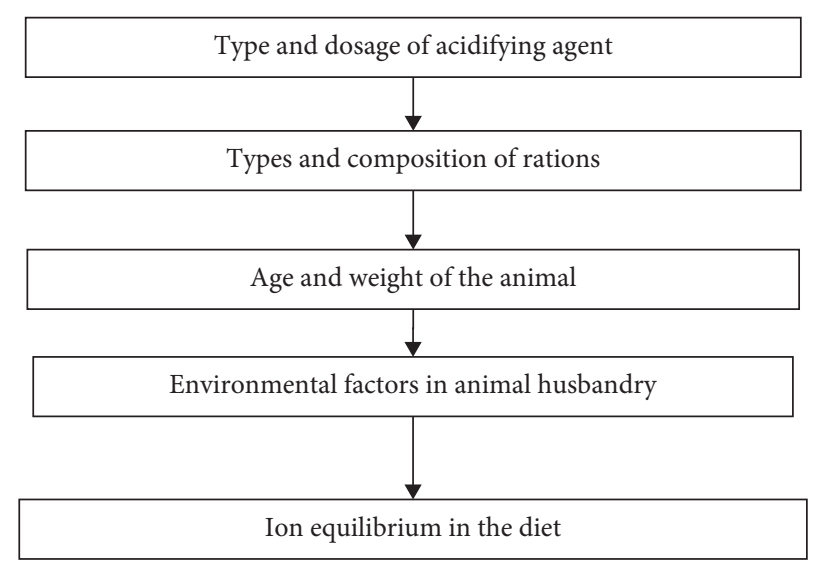

Figure 1: Flow charts affecting acidification effects.

Korsten et al. added $1.6 \%$ calcium formate in the feed to reduce the $\mathrm{pH}$ value of the feed by 0.6 and added $1 \%$ calcium formate and $0.5 \%$ propionic acid to reduce the $\mathrm{pH}$ value of the feed by 0.7 [5]. Flores et al. added $1.5 \%$ citric acid and $1.6 \%$ fumaric acid to the diet of piglets and reduced the dietary $\mathrm{pH}$ from 6.32 to 4.82 and 4.73 and the gastric contents from 4.33 to 3.86 and 3.87 , respectively [6]. It can be seen that acidifying agents can reduce the $\mathrm{pH}$ of stomach contents to some extent. Each enzyme has an appropriate $\mathrm{pH}$ environment, and the appropriate $\mathrm{pH}$ for pepsin is 2.2-3.6. The activity of pepsin decreased significantly when $\mathrm{pH}$ was higher than 3.7 and was inactivated when $\mathrm{pH}$ was higher than 6.0.

\section{Research on Acidifier}

\subsection{Classification of Acidizing Agents}

2.1.1. Inorganic Acidifier. Inorganic acidifiers usually include $\mathrm{H}_{3} \mathrm{PO}_{4}, \mathrm{HCl}$, and $\mathrm{H}_{2} \mathrm{SO}_{4}$, have strong sour taste and large output, and are easy to promote and use. Tests show that $\mathrm{H}_{2} \mathrm{SO}_{4}$ is essentially disabled. Due to different electrolysis conditions, $\mathrm{HCl}$ in use will affect the dietary effect. $\mathrm{H}_{3} \mathrm{PO}_{4}$ has the function of acidification, supplementing the mineral elements of phosphorus, and has a high cost performance, so it can be widely used in actual production. In short, inorganic acids acidify better than organic acids and have a price advantage. The disadvantage of inorganic acid is that it is difficult to grasp the reasonable amount of addition. Therefore, if the concentration is low, it will be invalid. If the concentration is high, it will damage the processing machine $[7,8]$.

2.1.2. Organic Acidifier. Common organic acidifying agents include formic acid, acetic acid, propionic acid, butyric acid, sorbic acid, fumaric acid, malic acid, tartaric acid, and citric acid [9]. Organic acidifiers are corrosive and expensive, but because of their taste, they often function in the body's tricarboxylic acid cycle, which promotes the growth and development of new animals, so feed companies give them priority.
2.1.3. Compound Acidifier. The use of organic or inorganic acidifiers alone has many advantages and disadvantages, and different reaction principles enable them to coordinate and complement each other. Artificial blending of two single acidifiers as complex acidifiers can provide rapid $\mathrm{pH}$ control, excellent buffering capacity, and higher cost performance, while reducing damage to equipment and machinery [10]. Further optimization of the mixing ratio to form mixed acidifiers is a general trend, and the appearance and application of odorous acidifiers in lactating cows is the best evidence. The mixed acidifying agent usually presents as powder, and the additive amount is $1.0-1.5 \mathrm{~kg} /$ feed [11].

Many acidifiers use white carbon black as the carrier and adsorb inorganic or organic acids. Silica itself has no nutritional value. The acidifier produced by a local research company is a compound organic acid obtained by direct enzymolysis of feed raw materials and fermentation. Its carrier is the raw material of fermentation feed and has high nutritional value, which is a breakthrough in the preparation of acidifier [12].

2.2. Factors Affecting Acidification Effect. In recent decades, many countries have imposed strict restrictions on the addition of antibiotics because the contamination of animal products with chemical residues of antibiotics is a serious threat to human health [13]. Acidifiers are becoming more and more popular as highly efficient, pollution-free, residuefree, and environmentally friendly feed additives, which can promote growth and inhibit the growth and reproduction of pathogenic microorganisms [14]. Because different acidifiers often have a synergistic effect on each other, a proportional mixing of different acidifiers can be more effective in preventing disease. Most complex acidifiers are based primarily on organic acids and are mixed with other organic acids. The use of compound acidification agents can increase the acidification effect of diet, significantly reduce the $\mathrm{pH}$ value of piglets' stomachs, and help to maintain the most suitable acidic environment in the digestive tract [15]. Most tests show that the combined acidifier is superior to the single acidifier. 
2.2.1. Type and Dosage of Acidizing Agent. There are many types of acidifiers, which have different molecular weights, solubility, dissociation constant, and energy value and have different effects. Insufficient dose cannot achieve the required acidification effect, and too much dose will affect the normal performance of animal production capacity.

2.2.2. Types and Composition of Meals. Different dietary types have different acidification effects.

2.2.3. Age and Weight of the Animal. Animal age and weight should be taken into account when adding acidifiers to feed. For example, after birth, the gastric acid secretion of piglets is insufficient, and digestive function is gradually improved with age and weight gain, gastric acid secretion is gradually increased, and acidification effect is decreased.

2.2.4. Environmental Factors for Raising Livestock. Hygiene, feed density, temperature, humidity, and various fence pressures also influence the effect of the acidifier.

2.2.5. Ion Balance in Diet. Adding inorganic or organic acidizing agents to the diet of animals often disrupts the ion balance of diet and leads to metabolic acidosis in animals [16].

Compound acidifier is composed of several specific proportions of single acidifier. According to the different composition, it can be divided into two categories: total acid compound acidifier and acid compound acidifier. All-acid compound acidifiers consist mainly of one acid and synergistic compound acidifier one or more other acids $[17,18]$. Currently, the most common acidifiers for all acid compounds are phosphoric acid and lactic acid. Phosphoric acid consists mainly of phosphoric acid and is mixed with other inorganic and organic acids. The lactic acid system consists mainly of lactic acid and is mixed with other inorganic and organic acids. Organic acid and organic acid salt form a composite acidulant through chemical coordination, which is the development trend of composite acidulant. Figure 1 shows a flow chart that affects the acidification effect.

\subsection{On the Role of Acidifier}

2.3.1. Action Mechanism of Acidifier. There are many kinds of acidizing agents used in fodder, and there are mainly three kinds in feed production, namely, inorganic acidifying agent, organic acidifying agent, and compound acidifying agent. The inorganic acids commonly used in production mainly include phosphoric acid, which is relatively cheap. The main organic acids are citric acid, fumaric acid, lactic acid, propionic acid, malic acid, sorbic acid, formic acid, and acetic acid. The compound acidifier can be divided into two kinds: the whole acid compound acidifier and the salt compound acidifier. The most common type of all-acid compound acidifier is phosphoric acid and lactic acid. The complex acidifier is a new generation of complex acid which is complexed by scientific proportion of organic acid and inorganic acid. It has strong acidity, low dose, fast action, and better effect than single acidifier. It is the tendency of feed acidifier application that the drug is safer and more effective. The action mechanism of acidifier is mainly reflected in the following aspects $[19,20]$ :

(1) Reduce the $p H$ Value of Feed, Reduce the $p H$ Value in the Stomach, and Improve the Activity of Digestive Enzymes. Adding acidifying agents to animal feed lowers the $\mathrm{pH}$ of the stomach, activates pepsin, and speeds up protein breakdown. Protein decomposition also stimulates the secretion of more trypsin in the duodenum, leading to complete protein degradation and absorption [21]. The gastric acid secretion of early weaning piglets is insufficient, the diet $\mathrm{pH}$ of piglets is between 5.8 and 6.5, and the gastric $\mathrm{pH}$ of piglets depends on PEPase, which is usually higher than the appropriate activity range. Absorption of organic acids in pig feed can increase the growth potential of piglets [22]. According to the experimental results in the literature, adding 1.6\% 2.1\% fumaric acid in the feed can increase the average daily weight gain of piglets by $9 \%$, feed intake by $5.2 \%$, and feed utilization rate by $4.4 \%$. The effect of adding hydrochloric acid and sulfuric acid into the diet of weaned piglets was studied in the experiment. The results showed that the intake quantity of weaned piglets decreased sharply, and the daily weight gain and the feed utilization rate decreased seriously.

(2) Slow Down the Rate of Gastric Emptying. The stimulating factors that promote the speed of digestive tract and gastric emptying are the volume and $\mathrm{pH}$ value of gastric contents [23]. After acidic chyme enters the small intestine, it stimulates the mucosa of the small intestine, making it secrete gastric inhibitory hormone, reflexivity inhibits gastric peristalsis, slows gastric emptying, and allows more time for protein digestion in the stomach [24].

(3) Improve Gastrointestinal Microflora. The suitable $\mathrm{pH}$ value for the growth of common pathogenic microorganisms in the gastrointestinal tract is neutral base. For example, the suitable $\mathrm{pH}$ value of Escherichia coli is 6.2 8.2, Streptococcus 6.1 7.5, and Staphylococcus 6.7 7.6, and lactic acid bacteria and other beneficial bacteria are suitable for growth and proliferation in an acidic environment [25]. Therefore, acidifying agents can inhibit the proliferation of harmful microorganisms and promote the proliferation of beneficial bacteria by reducing the $\mathrm{pH}$ value of the gastrointestinal tract. The metabolite of lactic acid bacteria (lactic acid) can block the binding of E. coli to its receptor in the intestine and inhibit the growth of $E$. coli. The main cause of diarrhea in weaned piglets is the increase in $\mathrm{pH}$ value in the gastrointestinal tract due to insufficient gastric acid secretion, which provides a suitable environment for the mass reproduction of E. coli. Within 2 days after weaning of piglets, Lactobacillus in ileum almost drops to zero, the $\mathrm{pH}$ value in ileum increases, and the mass reproduction of $E$. coli leads to diarrhea in piglets. The frequency of diarrhea can be significantly reduced by adding acidification agent into piglets' 
feed. Foreign studies have shown that organic acids entering the intestinal tract can change the intestinal morphology, such as weaned piglet's small intestinal crypt depth becoming shallow and increasing villi height.

(4) Promote the Absorption of Minerals and Vitamins. Some major and trace elements in the alkaline environment are easy to form insoluble salt and very difficult to absorb. Acidifier can not only reduce the $\mathrm{pH}$ value of gastrointestinal contents but also form complex which can be easily absorbed and utilized with some mineral elements. Many scholars have proved that the addition effect of high copper and acidifying agent has an additive effect; that is, fumaric acid, citric acid or phosphoric acid, and copper form a complex with high biological potency, which promotes the absorption and retention of copper and reduces the oxidation catalytic activity of copper at the same time.

Some organic acids, such as fumaric acid, have antioxidant effects, and citric acid is an antioxidant booster. When fumaric acid was added to premix and kept for 6 months, the stability of vitamin A and vitamin $\mathrm{C}$ was improved compared with that without fumaric acid. The acidic environment of the small intestine also facilitates the absorption of vitamins $\mathrm{A}$ and $\mathrm{D}$.

(5) Directly Participating in Energy Metabolism in the Body. As an energy source, some organic acids are important intermediates in the process of energy conversion and can be directly involved in metabolism. For example, the triclosate cycle reaction is initiated by the condensation of acetyl and oxaloacetate to citric acid; fumaric acid is also an intermediate product of the triclosate cycle; and lactic acid is also involved in metabolism in the body and is one of the end products of glycolysis. It release energy, so organic acid can be used as energy source.

\subsubsection{Action Mechanism of Composite Acidifier}

(1) Adjust the Balance of Microbial Flora in the Digestive Tract. For the growth of the most harmful bacteria in the gastrointestinal tract, the optimum acidity and alkalinity are neutral slants. E. coli, for example, has a suitable $\mathrm{pH}$ of $6-8$, while beneficial bacteria such as Lactobacillus are adapted to grow and reproduce in acidic environments. When acidifying agents enter the digestive tract of livestock and poultry, lowering the $\mathrm{pH}$ value can promote the growth and reproduction of beneficial bacteria (such as lactobacillus) and change the living environment of harmful bacteria (such as E. coli and Salmonella) and can control or kill Staphylococcus, shuttles, Bacillus, and other harmful bacteria.

(2) Reduce the pH Value of Digestive Tract and Enhance the Activity of Digestive Enzymes. Studies have shown that normal digestive physiology can be maintained only when the $\mathrm{pH}$ of the animal gastrointestinal tract is stable in a reasonable range. If the $\mathrm{pH}$ value is too high, pepsinogen activation will be severely impaired. Pepsin is inactivated at $\mathrm{pH}$ above 6 . In the early stages of weaning, the digestive and immune systems of
Table 1: Application experimental data of BFA on fat pigs.

\begin{tabular}{lcc}
\hline & Daily gain & Feed efficiency \\
\hline Pig feed & $21 \%-26 \%$ & $9 \%-14 \%$ \\
Extensive conditions & $33 \%-56 \%$ & $22 \%-36 \%$ \\
\hline
\end{tabular}

piglets are immature. During the period between breastfeeding and feed feeding, the production of acid in the esophagus and gastrointestinal tract of piglets is insufficient, making it difficult for the stomach of adult pigs to become acidic ( $\mathrm{pH} 2.0-3.5$ ). The addition of acidifying agent can reduce the $\mathrm{pH}$ value of stomach of young animals, provide a reasonable environment for digestion of animals, activate pepsin to maintain high activity, help to soften feed and decompose nutrients, help to cause disease, and prevent microbial digestive tract. The external environment enters the animal body and stimulates the duodenum to secret more trypsin, which leads to the complete protein absorption and the improvement in feed utilization. Citric acid in the feed is very effective: it is directly involved in the body's metabolism and promotes the absorption of nutrients. Some trace elements in weak or alkaline environment tend to form insoluble acids and difficult to absorb in livestock and poultry. When the acidifier is added to the feed, the trace element salt is easily decomposed and the complex with the acidifier is easily formed and then absorbed. Many scholars have demonstrated that the effects of adding high copper and acidifying agents have additive effects, such as increasing copper's oxidative catalytic activity, stress resistance, and animal immunity. Some studies have found that piglet feed has the highest nitrogen content of solid nitrogen fertilizer. The fertilizer is a neutral nitrogen fertilizer, has relatively stable physical and chemical properties and low hygroscopicity, and is easily soluble in water. When fertilized in soil, fertilizers are less efficient because they must be converted into bicarbonate and absorbed in large quantities into crops.

2.4. Mechanism ofBFA. The biochemical fulvic acid (BFA) is an active substance that simulates the natural humic acid formation environment and is fermented by organic materials such as crop straws. Due to its small molecular weight, it is easy to absorb and use in animals and has functions such as regulating physiological functions, nutritional management, disease prevention, and livestock treatment and is widely used in livestock and veterinary clinics.

2.4.1. To Promote Growth, Can Be Used as a Growth Accelerator. One is that nucleic acids, amino acids, vitamins, inositol, polysaccharides, and other substances found in biochemical fulvic acid can directly participate in human metabolism, which is an excellent nutrient and growth hormone in livestock and poultry. Second, nutrients are completely transformed into small molecular nutrients to promote the absorption of metabolites and improve productivity; thirdly, biochemical fulvic acid contains a quinone group, which is involved in the REDOX reaction of human body, maintains a strong metabolism, and promotes cell division and growth. Fourthly, biochemical fulvic acid ACTS on the vegetative 


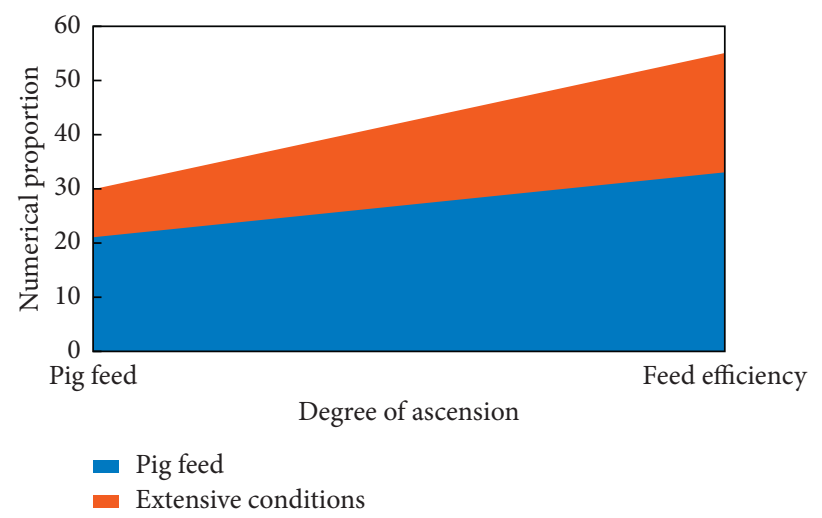

Figure 2: Application experimental data of BFA on fat pigs.

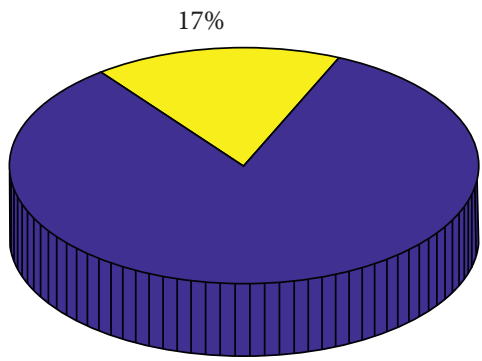

$83 \%$

Recovery rate

$\square$ Failure rate

FIgURE 3: Data on cure rates.

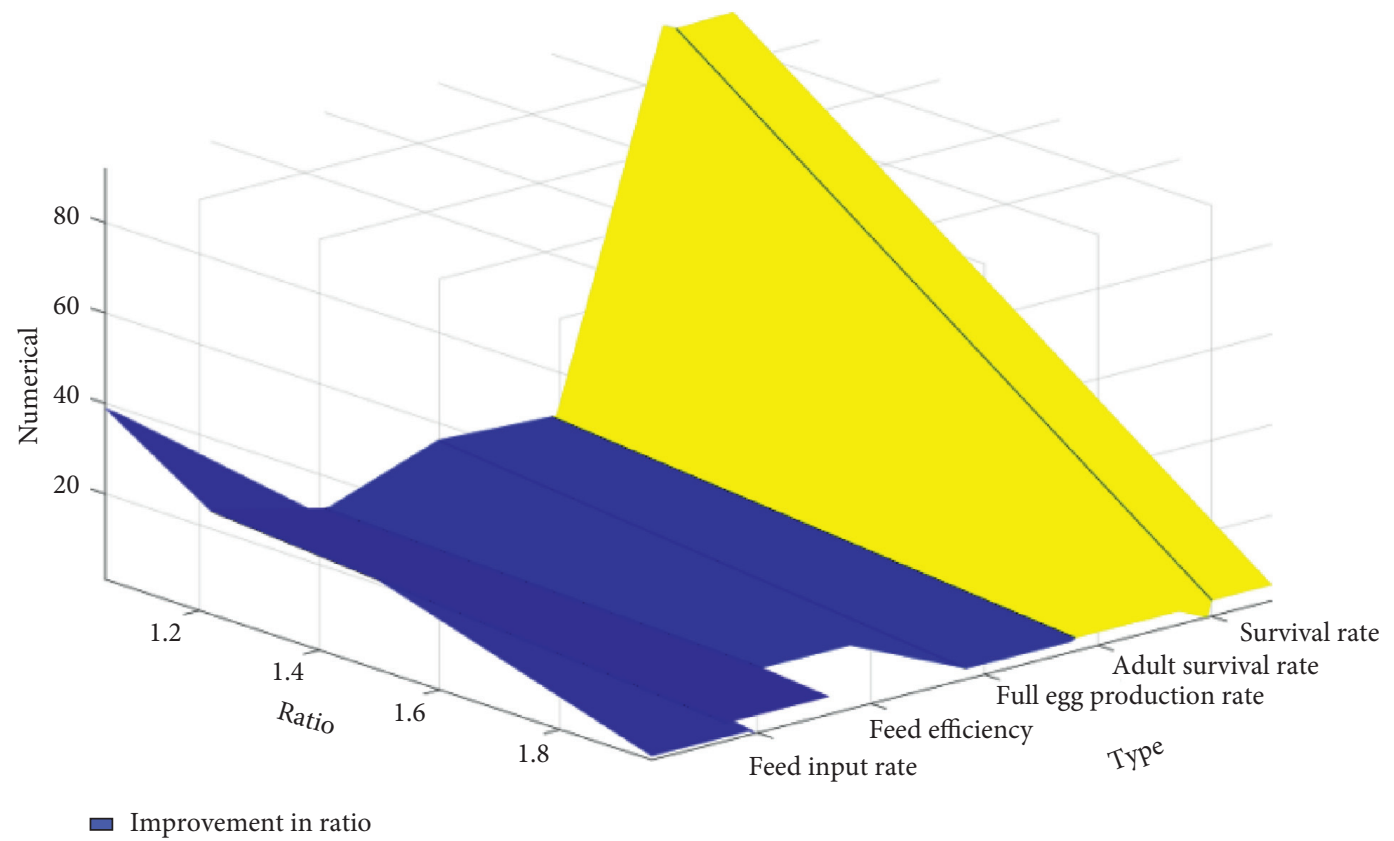

FIgURE 4: Specific data of BFA preparation added in feed. 
TABLE 2: Blood analysis specific data.

\begin{tabular}{lc}
\hline & Numerical \\
\hline Neutrophils & $7.21 \%-21.34 \%$ \\
Lymphocyte & $14.3-14.65 \%$ \\
Newcastle disease antibody & $16.18-33.23 \%$ \\
\hline
\end{tabular}

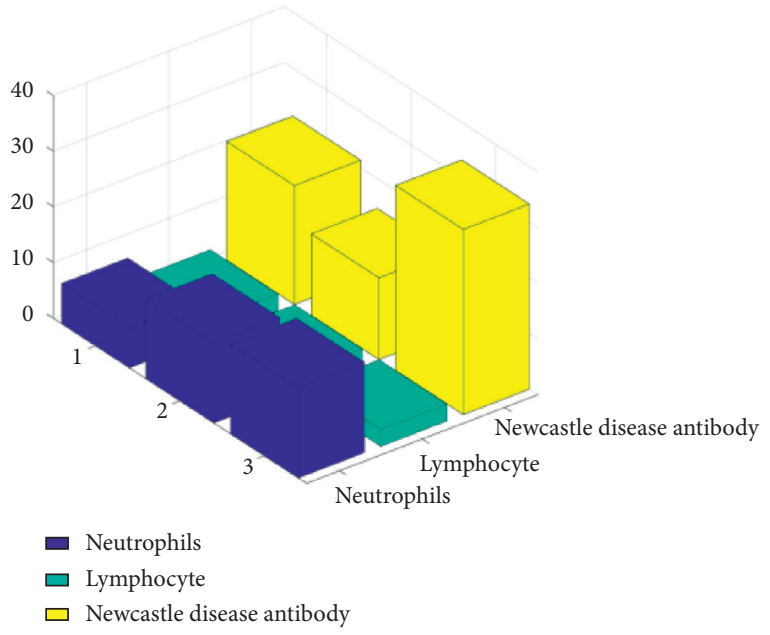

FIgure 5: Blood analysis specific data.

nervous system inhibits sympathetic excitation and slows down the heartbeat. Increased intestinal activity increases the content of digestive juices, reduces body temperature and consumption, and keeps livestock and poultry quiet, thereby improving feed use. Fifthly, biochemical fulvic acid is produced by fermentation of various common microbial strains, which contain various active enzymes, which can effectively promote the digestion and metabolism of livestock and poultry and reduce feed remuneration.

\subsubsection{To Prevent and Treat Diseases and Enhance Immune} Function. First of all, biochemical fulvic acid can cause human body to produce interferon, activate reticuloendothelium system, enhance nonspecific immunity, and has a strong resistance to pathogenic microorganisms; second, biochemical fulvic acid can activate monocyte system to increase cell number, phagocytic ability, and phagocytic activity and has an infectious stimulation effect on thymus, which can improve antistress ability and viral bacterial disease; third, its strong adsorption ability can effectively absorb feed and digestive tract and metabolic process and adsorb a variety of toxic and harmful substances; fourth, biochemical fulvic acid is an organic acid preparation, which can effectively regulate the $\mathrm{pH}$ value of the digestive tract.

\section{Experimental Study on Acidifier}

3.1. Selection of Experimental Materials. The addition of organic acids to a simple corn and soybean meal diet significantly improved piglets' daily weight gain, but the acidification effect of adding a complex diet containing dairy products remains unclear. Minerals and high protein diets have strong acid binding abilities. Compared with grain feed, it has a lower $\mathrm{pH}$ required for digestion. A diet high in calcium carbonate $(59 \mathrm{~g} / \mathrm{kg} \mathrm{DM})$ almost completely prevents bell-shaped acidification in the stomach. Diets with high CP (>30\%) did not increase piglets' daily weight gain, whereas diets with low CP (18\%) did.

3.2. Selection of Experimental Acidifier. In this study, the effect of acidifying agent was obvious in the first 1 3 weeks after early weaning of piglets, gradually decreased after 3 weeks, and basically had no effect after 4 weeks. Therefore, choosing an acidulant should be based on the age and weight of the animal.

3.3. Experimental Environment Setting. The results show that the combined use of organic acids and antimicrobials is usually better than the single use. In addition, the combination of organic acids, antibiotics, and high copper has the best effect. The three have different functions and have complementary or additive effects. The effect of acidifier is better than that of acidifier in the condition of poor environment.

\section{Application of Compound Chemical Treatment in Animal Husbandry}

\subsection{Application of Compound Chemical Treatment in Raising Pigs and Cows}

(1) The application of BFA preparation in porcine breeding. According to the experiments of Supervisor $\mathrm{Gu}$ Zilin and others in many pig farms, adding $0.6-1.2 \%$ pig feed can increase daily weight gain by $21 \%-26 \%$ and feed utilization rate by $9 \%-$ $14 \%$; under the extensive condition, the daily gain was increased by $33 \%-56 \%$, the feed utilization rate was increased by $22 \%-36 \%$, and the feed meat ratio was $3.45: 1-3.2: 1.0 .12 \mathrm{~kg}$ less material consumption per kg weight gain than the control group. BFA has the functions of preventing gastroenteritis, piglet diarrhea, and white myopathy. Long-term application of BFA in breeder pigs can improve the lactation rate and pregnancy rate of sows and improve semen level. The experimental data of application of BFA in fattened pigs are shown in Table 1 and Figure 2.

(2) Adding 1\% of BFA into the feed of dairy cows can increase the milk yield of dairy cows by $9-17 \%$ and the special milk yield by $19.12 \%$. It increases price of feed, lowers digestive tract disease, promotes the cows in the rumen microbial growth, accelerates the speed of rumen microbial protein synthesis, increases animal body by feed protein and protein nitrogen utilization, prevents metabolic disease, reduces postpartum reproductive system disease, prolongs peak milk production, and maintains high ability of cattle of sexual reproduction rate and high yields. Prevention and treatment of dairy 
cow mastitis has special effects, among which the effective rate of acute mastitis is $100 \%$, the cure rate is $83 \%$, the recessive mastitis can be controlled below $13 \%$, the clinical mastitis can be reduced by 1.8-2.1 percentage points, and the alcohol-positive milk can be prevented. Fulvic acid can cause dairy cow milk somatic cells to decrease for a short time, and then the immune function is activated very quickly; the performance is increased lymphocytes in the blood because the somatic cells move to the breast in large quantities, so the somatic cells in milk also increase, thus improving the cellular immunity of the body. Among them, the highly functional phagocytes also remove the causes of mastitis so that mastitis can be cured. Combined with antibiotics, mastitis has a better effect without any toxic and side effects and has antistress so that the milk yield is relatively stable and the cure rate is shown in Figure 3.

\subsection{Application of Compound Chemical Treatment in Raising Chickens}

(1) Adding 1.3\% BFA preparation into chicken feed can increase the growth rate and improve the uniformity of chicken and improve the specific and nonspecific immunity of chicks. The survival rate is as high as $97 \%-99.2 \%$. The survival rate of laying hens was $97 \%-100 \%$ when $1.5 \%$ BFA was added into the feed. In addition, the egg production period is advanced by 4-6 days, and the egg production peak (egg production rate is above 90\%) can be extended by more than two months. The egg production rate increased by $12.3 \%$, the feed utilization rate increased by $13.58 \%$, and the feed input decreased by $4.61 \%$. Compared with the control group, the strength and thickness of the eggshell increased by $10.7 \%-15.8 \%$ and $5.9 \%-11.2 \%$, respectively. The thickness of the sponge layer of the eggshell also increased significantly, and the soft-broken egg rate decreased by $41.6 \%$. $2 \%$ BFA preparation was added into the feed of broiler chickens, and it was confirmed that the feed utilization rate was increased by $8.1 \%$ and $0.192 \mathrm{~kg}$ of feed was saved per $\mathrm{kg}$ of weight gain. The meat ratio of 7 -week-old broiler is $2.2-2.26: 1$. Group dosing decreased by $26 \%$, reduced drug retention, and improved meat quality. The specific data of ADDING BFA in feed are shown in Figure 4.

(2) Blood analysis showed that the antibody values of neutrophils, lymphocytes, and Newcastle disease increased by $7.21-21.34 \%, \quad 14.3-14.65 \%$, and $16.18-33.23 \%$, respectively. The maintenance time of antibody peak increased by 3-7 days. For infectious bronchitis, laryngotracheitis, pullorosis, coccidiosis, and Salmonellosis, it has good prevention and control effect. Specific data of blood analysis are shown in Table 2 and Figure 5.

\section{Conclusions}

(1) Biochemical fulvic acid (BFA) is not only a mixed nutritional product of microbial metabolites but also an antibiotic-based drug. Its application performance is basically stable, and the effect is even more stable than antibiotic. With the growing demand for green, pollution-free meat, eggs, and milk, the country has set strict quality standards for staple foods entering the market, causing serious problems for the feed industry. There is an urgent need to "people-oriented" and produce new pollution-free green products. So far, some feed producers have unilaterally pursued feed "effects" against the perverse psychology of some farmers. In the production of feed, regardless of the state of health of the consumer, large amounts of antibiotics must be added, and only the growth and production of livestock and poultry is sought. In order to achieve the purpose of production, many feed producers add chemical elements that have toxic and side effects to humans and animals, high residues of chemical elements, adrenaline, and diazepam. Prolonged use of antibiotics in feed not only destroys the normal microbial system of the gastrointestinal tract but also accumulates residual drugs and strains in animals, increasing drug resistance and increasing drug use. The increase and effectiveness of drugs decrease year by year. In recent years, some probiotics, oligosaccharides, enzyme preparations, and saponins have emerged, but due to poor stability or inconsistent efficacy, they cannot completely replace antibiotics. With the advent of BFA, a new green feed additive has been provided for harmless livestock and poultry breeding as well as alternative feed, thus opening up a wide range of potential applications in animal husbandry.

(2) Due to the various functional groups and active factors contained in biochemical fulvic acid, it exhibits various unique physical and chemical properties and can perform various physiological functions after entering the bodies of plants and animals. By inhibiting or activating this enzyme, it acts on the metabolism of animals and plants, reflects a significant stimulating effect, and plays a therapeutic role by regulating endocrine hormones and improving the immune function of the human body. Due to its easy bioabsorption and strong biological activity, biochemical fulvic acid has many uses and application effects in animal husbandry, veterinary medicine, and food, but it is the best dose for special animals. As further studies have not yet been undertaken, studies in determining yield are of great development value.

(3) The research results of this paper show that adding $1 \%$ of BFA into cow feed can increase the milk yield of primary milk by $9-17 \%$ and the milk yield by $19.12 \%$. Biochemical fulvic acid is a kind of green physiological active substance, which is obtained by fermenting special microorganisms with straw and 
other organic matter. Through the research results, it is found that it has the function of promoting the growth of animals and plants and enhancing immunity and has been widely used in ruminants, monogastric animals, and aquatic animals in recent years.

\section{Data Availability}

No data were used to support the findings of the study.

\section{Conflicts of Interest}

The authors declare that there are no conflicts of interest regarding this submission.

\section{Acknowledgments}

This study was supported by the pillar Project of Hunan Vocational College of Environmental Biological Technology.

\section{References}

[1] L. Ayed, N. Asses, N. Chammem, and M. Hamdi, "Improvement of green table olive processing wastewater decolorization by Geotrichum candidum," Desalination and Water Treatment, vol. 57, no. 37, pp. 17322-17332, 2015.

[2] Z. Liu, X. Li, F. Hu, and P. Zhan, "Oxidation performance of $\mathrm{FeO} / \mathrm{Na}_{2} \mathrm{~S}_{2} \mathrm{O}_{8}$ process for the treatment of landfill leachate biochemical effluent as well as activator characterization," Desalination and Water Treatment, vol. 107, pp. 109-117, 2018.

[3] B. Elzen and B. Bos, "The RIO approach: design and anchoring of sustainable animal husbandry systems," Technological Forecasting and Social Change, vol. 145, pp. 141-152, 2019.

[4] A. Eicker, E. Forootan, A. Springer, J. Kusche, and L. Longuevergne, "Does GRACE see the terrestrial water cycle "intensifying"?" Journal of Geophysical Research: Atmospheres, vol. 121, no. 2, pp. 733-745, 2016.

[5] H. Korsten, A. C. J. Z. V. D. Made, W. M. V. Weerden et al., "Characterization of heterogeneous prostate tumors in targeted pten knockout mice," PLoS One, vol. 11, no. 1, Article ID e0147500, 2016.

[6] A. L. Flores, K. Risley, J. Zanoni et al., "Factors of success for transitioning from a scientific role to a supervisory leadership role in a federal public health agency, 2016," Public Health Reports, vol. 134, no. 5, pp. 466-471, 2019.

[7] M. K. Gecer, M. Akin, M. Gundogdu, S. P. Eyduran, S. Ercisli, and E. Eyduran, "Organic acids, sugars, phenolic compounds, and some horticultural characteristics of black and white mulberry accessions from Eastern Anatolia," Canadian Journal of Plant Science, vol. 96, no. 1, pp. 27-33, 2016.

[8] H. Zheng, Q. Zhang, J. Quan, Q. Zheng, and W. Xi, "Determination of sugars, organic acids, aroma components, and carotenoids in grapefruit pulps," Food Chemistry, vol. 205, pp. 112-121, 2016.

[9] T. S. Anirudhan, F. Shainy, and J. Christa, "Synthesis and characterization of polyacrylic acid- grafted-carboxylic graphene/titanium nanotube composite for the effective removal of enrofloxacin from aqueous solutions: adsorption and photocatalytic degradation studies," Journal of Hazardous Materials, vol. 324, pp. 117-130, 2016.
[10] B. Qin, G. Dou, Y. Wang, H. Xin, L. Ma, and D. Wang, “A superabsorbent hydrogel-ascorbic acid composite inhibitor for the suppression of coal oxidation," Fuel, vol. 190, pp. 129-135, 2017.

[11] D. Li, Y. Jiang, S. Lv et al., "Preparation of plasticized poly (lactic acid) and its influence on the properties of composite materials," PLoS One, vol. 13, no. 3, Article ID e0193520, 2018.

[12] L. Zhu, Y. Li, C. A. Carrera, Y-C. Chen, M. Li, and A. Fok, "Calibration of a lactic-acid model for simulating biofilminduced degradation of the dentin-composite interface," Dental Materials, vol. 33, no. 11, pp. 1315-1323, 2017.

[13] V. G. Goffman, V. V. Sleptsov, N. N. Kovyneva, N. V. Gorshkov, O. S. Telegina, and A. V. Gorokhovsky, "Effect of nanosized potassium polytitanate on the properties of proton-conducting composite based on phosphotungstic acid and polyvinyl alcohol," Theoretical and Experimental Chemistry, vol. 52, no. 5, pp. 1-5, 2016.

[14] H. Zhang, J. Zhu, X. Cheng et al., "Effect of modified vermiculite on the interface of a capric acid-expanded vermiculite composite phase change material with phase transition kinetics," Journal of Wuhan University of Technology-Materials Science Edition, vol. 34, no. 2, pp. 345-352, 2019.

[15] X. Zhang and Y. Xu, "The fabrication of polypyrrole/ D-tartaric acid composite used as electrode in supercapacitors," Materials Letters, vol. 190, pp. 240-243, 2017.

[16] F. Lynda, B. Tanushree, N. Powe, and A. Sebastian, "Acid balance, dietary acid load, and bone effects-a controversial subject," Nutrients, vol. 10, no. 4, p. 517, 2018.

[17] J.-H. Lee, H. H. Kim, Y. H. Cho, T.-S. Koo, and G. W. Lee, "Development and evaluation of raloxifene-hydrochlorideloaded supersaturatable SMEDDS containing an acidifier," Pharmaceutics, vol. 10, no. 3, p. 78, 2018.

[18] A. Septembre-Malaterre, F. Remize, and P. Poucheret, "Fruits and vegetables, as a source of nutritional compounds and phytochemicals: changes in bioactive compounds during lactic fermentation," Food Research International, vol. 104, pp. 86-99, 2018.

[19] H. E. E. Malik, R. H. H. Hafzalla, O. H. A. Ali et al., "Effect of probiotics and acidifiers on carcass yield, internal organs, cuts and meat to bone ratio of broiler chicken," Journal of Agriculture and Veterinary Sciences, vol. 9, no. 12, pp. 18-23, 2016.

[20] J. Wang, J. Yuan, K. W. Kim, and F. Xiao, "Chemical, thermal and rheological characteristics of composite polymerized asphalts," Fuel, vol. 227, pp. 289-299, 2018.

[21] Y. Zhang, X. Rui, J. Wei et al., "Wet-chemical processing of phosphorus composite nanosheets for high-rate and highcapacity lithium-ion batteries," Advanced Energy Materials, vol. 6, no. 10, Article ID 1502409, 2016.

[22] M. Niu, W. Xu, S. Zhu et al., "Synthesis of nanoporous $\mathrm{CuO} /$ $\mathrm{TiO}_{2} / \mathrm{Pd}-\mathrm{NiO}$ composite catalysts by chemical dealloying and their performance for methanol and ethanol electro-oxidation," Journal of Power Sources, vol. 362, pp. 10-19, 2017.

[23] O. Budnik, A. Budnik, K. Berladir, V. Sviderskiy, and P. Rudenko, "Structural conformation of polytetrafluoroethylene composite matrix," Chemistry \& Chemical Technology, vol. 10, no. 2, pp. 241-246, 2016.

[24] Y. Liang, H. Ding, and Q. Xue, "Characterization of brucite/ $\mathrm{TiO}_{2}$ composite particle material prepared by mechanochemical method," Surface Review and Letters, vol. 25, no. 4, p. 1132, 2018.

[25] M. Zatoń, J. Rozière, and D. Jones, "Mitigation of fuel cell PFSA membrane chemical degradation using composite cerium oxide-PFSA nanofibres," Journal of Materials Chemistry A, vol. 5, no. 11, pp. 5390-5401, 2017. 